\title{
GAIA Level 1 Preeclampsia with Severe Features
}

National Cancer Institute

\section{Source}

National Cancer Institute. GAlA Level 1 Preeclampsia with Severe Features. NCI

Thesaurus. Code C128006.

GAIA Level 1 Preeclampsia with Severe Features is defined by three criteria: first, the gestational age of the pregnancy must be greater than or equal to 20 weeks; second, a diagnosis of new onset maternal hypertension (systolic blood pressure greater than or equal to $140 \mathrm{mmHg}$ and/or diastolic blood pressure greater than or equal to $90 \mathrm{mmHg}$ ) that is sustained on two measurements over a minimum of one hour); third, the presence of one or more of the following requirements: a) Systolic blood pressure greater than or equal to $160 \mathrm{mmHg}$ and/or diastolic blood pressure greater than or equal to $110 \mathrm{mmHg}$, which is confirmed after only minutes; b) Development of severe, persistent headache; c) Development of visual changes; d) Eclampsia; e) New onset thrombocytopenia (platelets less than 100,000/microliter); f) New onset unremitting epigastric pain; g) AST and ALT elevated to twice the upper limit of normal; h) Evidence of liver capsular hematoma or liver rupture (diagnosed on clinical exam or with imag ing); i) Worsening renal function, as evidenced by serum creatinine level greater than $1.1 \mathrm{mg} / \mathrm{dL}$, a doubling of the serum creatinine (absent other renal disease), or oliguria (less than 500 cc/24 hours); j) Pulmonary edema (confirmed on either imaging with chest $x$-ray or on clinical exam). 